\title{
Peripheral vein detection using electrical impedance method
}

\author{
M. B. Al-Harosh ${ }^{1,2}$ and S. I. Shchukin ${ }^{1}$ \\ 1. Bauman Moscow State Technical University, Moscow, Russia \\ 2. E-mail any correspondence to: mujeebbajjash@mail.ru
}

\begin{abstract}
The electrical impedance method of peripheral vein detection is a novel approach, which offers the advantages of not being expensive and the capability of minimizing and reducing the difficulty of achieving intravenous access in many patients, especially pediatric and obese patients. The electrical impedance method of peripheral vein detection is based on the measurement of electrical impedance using the 4-electrode technique by applying a known alternating current of frequency $100 \mathrm{kHz}$ and constant amplitude to a set of current electrodes and measuring the resulting surface potential at two separate electrodes. This paper presents the results of investigations to estimate the efficiency of this method.
\end{abstract}

Keywords: Electrical impedance, venous occlusion, vein diameter, electrode system, vein projection on skin surface

\section{Introduction \\ In most situations, diagnosis or treatment of medical conditions require blood draw or drug and fluid administration, which can be achieved through proper venous access in the patients. According to a recent study, it has been estimated that there are nearly 500 million venipunctures done every year. Other studies have shown that 95.2-97.3 percent of them are successful in the first attempt, which indicates that it is difficult to find veins in around 14 million cases on the first try. In addition, 15,000 patients per day are subject to four or more attempts to draw blood or other fluids from the vein causing them to experience discomfort and pain [1]. To gain intravenous access, traditionally a clearly visible and palpable vein is used, but for many reasons such as: the patient's obesity, decreased venous tone, small diameter of veins, poorly contoured or moving vein, abnormal location of the vein,}

and the nursing staff skill - there is a possibility of unsuccessful punctures and injury. Development of vein visualization technology is particularly useful in such cases. Several devices have been developed recently to aid physicians, phlebotomists and surgeons in finding veins to avoid unnecessary sticks. A few such devices work by illuminating the patient's skin with near-infrared light (wavelength typically ranges from 700-1000 nm) showing peripheral veins for access. High-resolution ultrasound scanners provide good quality images of the superficial and deep veins in obese patients and small veins in pediatric patients in real-time. Venography provides an image of the veins after the patient is injected with a contrast dye [1].

Each method, mentioned above, has its own advantages and disadvantages. The electrical impedance method has been suggested as an alternative method with the advantages of being non-invasive and relatively cheap [2]. This method involves applying alternating current to the region of interest, by direct injection via skin electrodes and extracting information about the inner bioelectrical properties distribution through the measurements of the developing surface electrical potentials due to the current flow [3].

The region of interest is the forearm, which consists of complex biological tissues such as muscle tissue, nerve, fat, connective tissue, skin, and blood vessel (fig.1). The biological tissues of the forearm have different physical properties and different resistivity. The electrical resistivity of venous blood is lower than the surrounding tissue, which makes it possible to detect veins by electrical impedance method. The analysis of the electrical properties of biological tissues at several excitations frequencies that has been 
considered in related works $[4,5]$ has shown that the difference between the electrical resistivity of blood and muscle tissue increases with decreasing frequency.

However, measurement at frequencies below 70-100 $\mathrm{kHz}$ can cause nerve and muscle stimulation. Hence, it was concluded that the most adequate frequency range is $80-$ $100 \mathrm{kHz}$. Thus, electrical current with $100 \mathrm{kHz}$ is commonly used for electrical impedance tomography. Alternating current of frequency $100 \mathrm{kHz}$ and constant amplitude of 3 $\mathrm{mA}$ has been used in our study.

Table 1 lists the electrical resistivity of forearm biological tissues at a frequency of $100 \mathrm{kHz}$ [6].

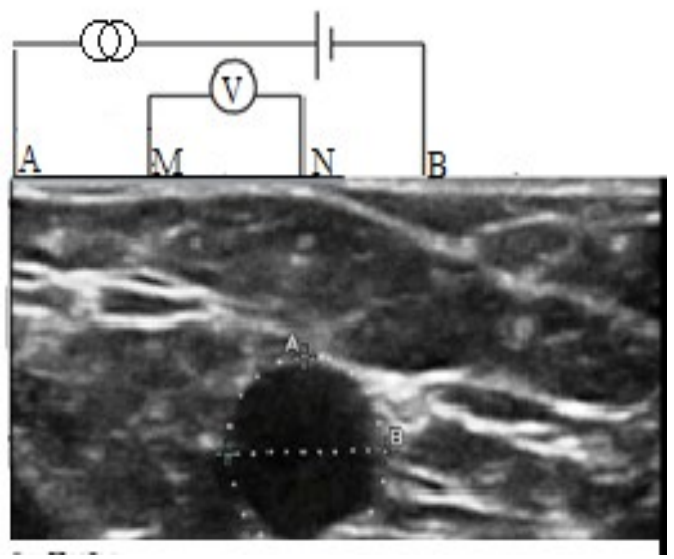

Fig. 1: Schematic drawing of the electrodes on the region of interest: $A, B$ are the current electrodes. $M, N$ are the potential electrodes.

Table 1: The electrical resistivity of forearm biological tissues.

\begin{tabular}{lc}
\hline Tissue & Electrical resistivity $\rho[\Omega \mathrm{m}]$ \\
\hline Muscle tissue & $2.76 \pm 0.3$ \\
Connective tissue & $2.5 \pm 0.5$ \\
Blood & $1.42 \pm 0.6$ \\
Nerve & $12.5 \pm 0.5$ \\
Subcutaneous fat & $25 \pm 0.7$ \\
Blood vessel wall & $3.13 \pm 0.2$
\end{tabular}

\section{Materials and methods}

To perform electrical impedance measurements, a special electrode system was designed to be attached to the study area. The electrode system, which is an array of surface electrodes, has four channels of tetra-polar electrode configurations. The electrode system has a distance between current electrodes $21 \mathrm{~mm}$ and $7 \mathrm{~mm}$ between potential electrodes. The measuring step is equal to the electrode diameter, which is $3.5 \mathrm{~mm}$. According to [7], this electrode system can provide visualization of vein projection on the skin surface of the forearm in a depth range of at least 6 and $9 \mathrm{~mm}$ for veins with diameter 2 and $5 \mathrm{~mm}$, respectively. However, due to the small surface of the current electrodes, it is requiring a long time to stabilize the transient resistance of the skin-electrode contact. The stability of the skin-electrode contact can also be achieved by pressing the electrode system to the region of interest, but pressing on the patient's skin with the electrode system can cause the vein to collapse. This is not desirable in such measurement, thus the collapsed vein cannot be detected.

This paper presents the new electrode system shown in figure 2. In this electrode system, the surface of the current electrodes has been increased and thus the stability of the skin-electrode contact improved so measurements were much more reliable and feasible for larger time periods [8].

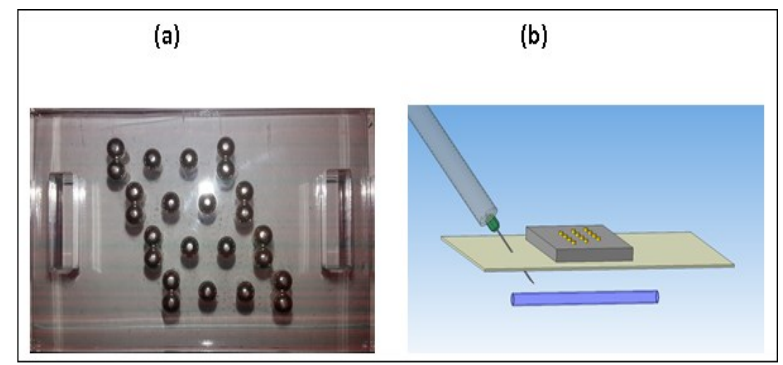

Fig. 2: a) The electrode system. b) The location of electrode system with respect to the vein.

The impedance was recorded with the multichannel system (REO-32) which has been developed at the Department of Medical and Technical Information Technology at Bauman Moscow State Technical University.

The measurements on participants have shown the limit of peripheral veins detection by the absolute impedance values. The main reason of limiting the use of this method on biological objects is that the influence of the inhomogeneity of the electro-physical properties of soft tissues on the measured impedance values, is comparable with the change due to blood vessel depth location. So factors affecting the absolute impedance are eliminated by only measuring relative impedance changes, rather than absolute impedance values. To display the relative impedance changes, venous occlusion has been used as a method of vein cross-sectional changes. The principle of the methodology is that the venous return from the forearm is briefly interrupted by inflating a cuff, placed around the upper arm, to well above venous pressure but below diastolic pressure $[9,10]$. The vein cross-sectional change has been estimated using an ultrasound system. Figure 3 shows how venous occlusion can be used to increase the crosssectional area of veins. The vein has been occluded to 60 $\mathrm{mm} \mathrm{Hg}$.

(a) (b) (c)

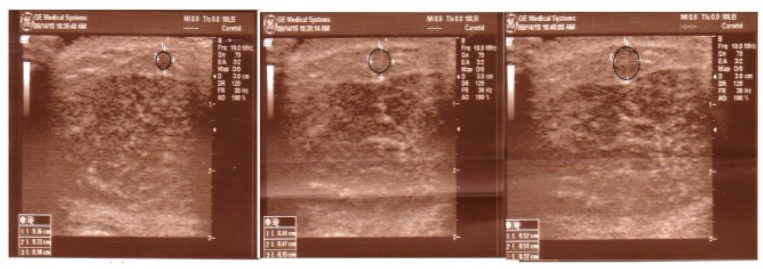

Fig. 3 The vein cross-sectional changes: a) The vein before occlusion. b) The vein after $30 \mathrm{sec}$ of occlusion. c) The vein after 1 minute of occlusion. 
Figure 4 shows the method of peripheral vein detection where the object of study was the forearm. To remove stratum corneum and increase conductivity, the skin is pretreated with alcohol. The electrode system was attached without using strong pressure to eliminate the possibility of blood vessel compression, the electrical impedance signal was recorded and simultaneously an inflation pressure of around $60-70 \mathrm{~mm} \mathrm{Hg}$ was used for intervals of $2 \mathrm{~min}$, and then the cuff is deflated. The recorded electrical impedance signal in figure 5 shows the process of vein cross-sectional changes as a function of electrical impedance changes. This signal was obtained by the channel that has identified the location of the vein. Hence, the vein is totally located between the measuring electrodes of this channel.

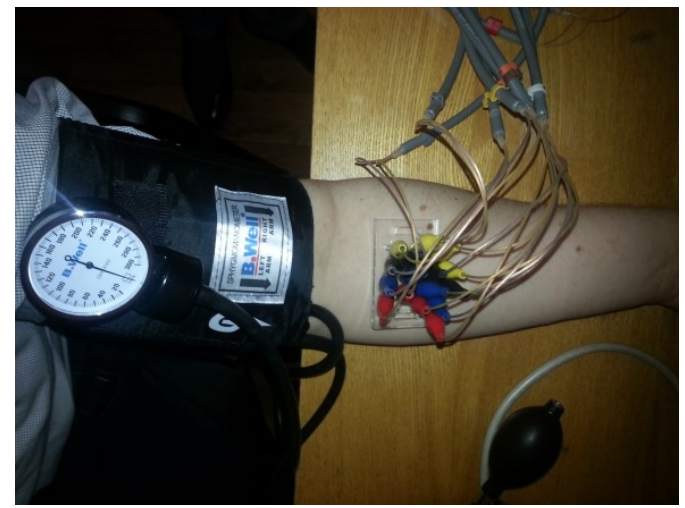

Fig. 4: The method of peripheral vein localization.

When the cuff pressure becomes close to the venous blood pressure, the vein is occluded and the blood outflow is stopped. This leads the vein diameter to increase, which corresponds to the decreasing in the recorded electrical impedance signal (phase 1). The phase 2 is associated with the event when the blood flow to the occluded vein leads to gradual increase in venous pressure. The increasing of venous pressure allows the collateral blood flow, which in turn stops the changes of vein diameter. When the cuff is deflated, the rate of venous outflow is proportional to the rate of decrease in volume; this corresponds to a decrease in vein diameter, which is reflected in the increase of the impedance signal (phase 3).

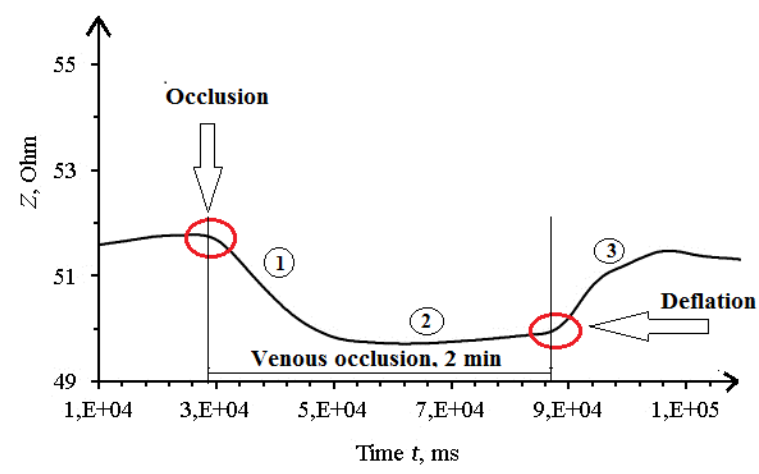

Fig. 5: The recorded electrical impedance signal.

\section{Results}

As a result of experimental studies on the patients without using the venous occlusion method, the impedance value is minimized in the localization of veins. For example, the first experiment on the first patient, which corresponds to the case when the vein is located between the measuring electrodes of the first channel, shows the minimum value of impedance as compared with other channels as shown in table 2. But the results of all measurements, recorded from 5 subjects, shows that the location of vein (marked green in table 2) was not corresponding to the minimum value of the impedance with all patients. Because of the inaccuracy of this method, a relative impedance changes has been used instead of absolute impedance values.

Table 2: The impedance values

\begin{tabular}{ccccc}
\hline \multirow{2}{*}{ Patient } & \multicolumn{4}{c}{ Absolute impedance values [S] } \\
& Channel 1 & Channel 2 & Channel 3 & Channel 4 \\
\hline 1 & 54.2 & 59.2 & 57.7 & 55.5 \\
2 & 45.7 & 49 & 46.3 & 47 \\
3 & 53.7 & 54.2 & 51.5 & 52.2 \\
4 & 88.5 & 81.9 & 81 & 78.8 \\
5 & 129 & 127.2 & 131.9 & 134 \\
\hline
\end{tabular}

Figure 6 shows that venous occlusion to $60 \mathrm{~mm} \mathrm{Hg}$ led to larger cross-sectional area of the veins. The cross-sectional area shape was calculated with the formula D1.D2. $\pi / 4$, where D1 and D2 are the maximum and minimum diameter of vein, respectively. The vein cross-sectional change was estimated using an ultrasound system for different depth of veins in soft tissues. The cross-sectional area increased from $9.3 \pm 2 \mathrm{~mm}^{2}$ to $20.8 \pm 2 \mathrm{~mm}^{2}$ for vein depth $1.8 \mathrm{~mm}$, from $2.1 \pm 1 \mathrm{~mm}^{2}$ to $3.8 \pm 1 \mathrm{~mm}^{2}$ for vein depth $0.7 \mathrm{~mm}$ and from $8 \pm 2 \mathrm{~mm}^{2}$ to $18.2 \pm 2 \mathrm{~mm}^{2}$ for vein depth $6.1 \mathrm{~mm}$. The maximum increase in cross sectional area was reached after 1 minute of occlusion.

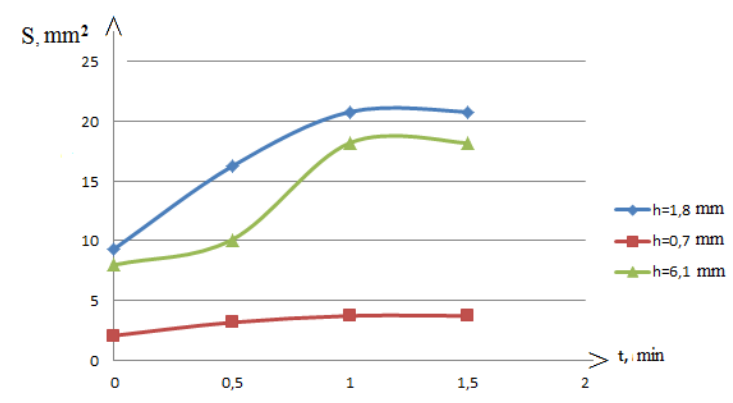

Fig. 6: The vein cross-sectional changes during occlusion.

Figure 7 shows the recorded electrical impedance signals during the procedure of vein detection. The detected vein has a diameter of $2.3 \mathrm{~mm}$ and a depth of $2 \mathrm{~mm}$. The first derivative of the signals has been used as an algorithm to detect the vein by the corresponding minimum peak during inflation and the maximum peak during deflation. 

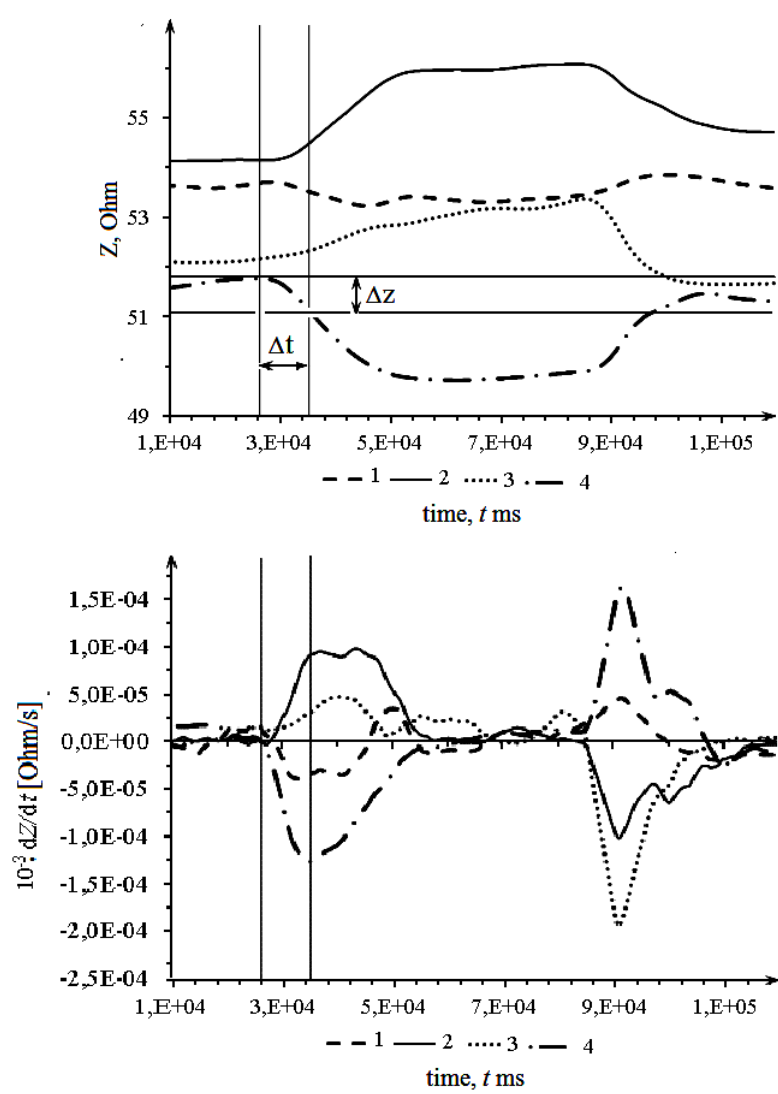

Fig. 7: The impedance signals obtained by electrode system.

The four electrical impedance signals obtained by the four-channel electrode system, shows different impedance changes against the location of vein regarding to each measuring channel. The more sensitive channel to high impedance changes identifies the location of vein as signal 4 in figure 7.

The inversion of impedance signals, which corresponds to impedance increasing during occlusion, was shown in some measuring channels such as signal 2 , due to vein location between the adjacent current and potential electrodes. The inversion effect disappears gradually with increasing vein depth. Figure 8 shows the analogous electrical impedance signals for vein with $2.8 \mathrm{~mm}$ diameter and a depth of $5 \mathrm{~mm}$.

As shown in figure 8 , the signal 1 is identifying the location of the vein. The inversion effect of impedance was not detected for this vein. According to a previous study, it has been estimated that for signals correspond to vein depth greater than $3 \mathrm{~mm}$ the effect of impedance inversion has disappeared [5]. On basis of the first derivative, the numerical characteristics such as the phase associated with the event of rapid vein filling $(\Delta \mathrm{Z})$ and the duration of the rapid vein filling $(\Delta t)$, have been identified to set the requirements to the procedure of vein detection. The Table 3 lists the numerical characteristics of impedance changes of the detected vein during the procedure of peripheral veins detection and the geometrical characteristics of veins such as diameter and depth. Thus, to reduce the time of achieving intravenous access, it is enough to detect veins after $30 \mathrm{sec}$ of venous occlusion.
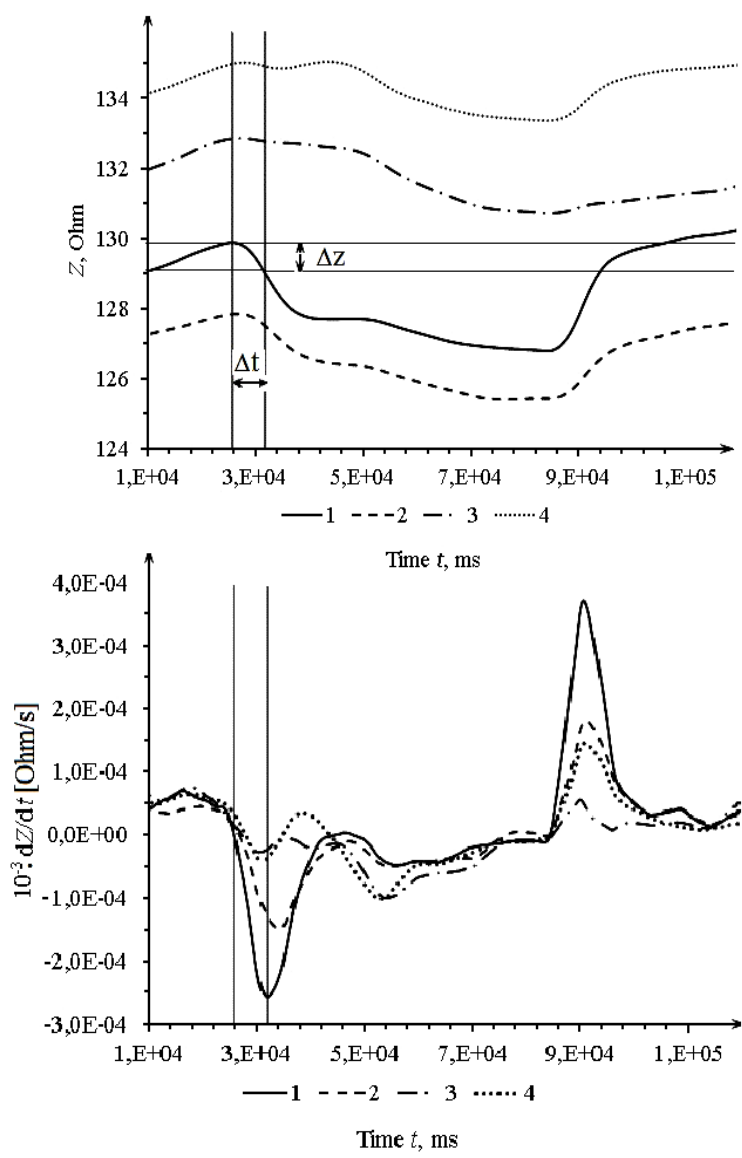

Fig. 8: The impedance signals obtained by electrode system.

Table 3: The numerical characteristics of the impedance changes.

\begin{tabular}{cccccc}
\hline Patient & $\boldsymbol{\Delta} \mathbf{Z}[\Omega]$ & $\boldsymbol{\Delta t}[\mathrm{sec}]$ & $\mathbf{h}[\mathrm{mm}]$ & $\mathbf{d}[\mathrm{mm}]$ & $\mathbf{Z}[\Omega]$ \\
\hline 1 & 0.68 & 17.8 & $1.8-2.2$ & $2.5-2.8$ & 44.3 \\
2 & 0.37 & 19.3 & $1.8-2.1$ & $2.3-2.5$ & 59.6 \\
3 & 0.86 & 12.7 & $4.9-5.2$ & $2.8-3.0$ & 132.3 \\
4 & 0.34 & 14.5 & $2.0-2.3$ & $2.2-2.5$ & 46.5 \\
5 & 0.68 & 8.6 & $3.2-3.5$ & $2.8-3.0$ & 88.9 \\
\hline
\end{tabular}

\section{Conclusions}

A new method of peripheral vein detection has been proposed. The method can identify vein projection on skin surface using a multichannel electrode system. The method of peripheral vein detection is based on regional monitoring the location of the vein in each channel of the electrode system after applying venous occlusion to $60 \mathrm{~mm} \mathrm{Hg}$ for 30 sec. The proposed method of peripheral vein detection can be used in such cases when the vein is not visible to the naked eye, especially with pediatric and obese patients. The electrical impedance method of peripheral vein detection determines the vein center projection on skin surface with an accuracy of a half electrode, which is $1.75 \mathrm{~mm}$.

Conflict of interest

The authors declare that they have no conflict of interest. 


\section{References}

1. Ganesh, Soujanya. Depth and size limits for the visibility of veins using the veinviewer imaging system. Diss. The University of Memphis, 2007.

2. Holder, D. S., and T. Tidswell. Electrical Impedance Tomography: Methods, History and Applications. CRC Press. Ed: DS Holder. (2004)

3. Zlochiver, Sharon. Induced current electrical impedance tomography for medical application a theoretical study. Diss. Tel-Aviv University, 2005

4. Kudashov I., S. Shchukin, O. Belaya, and S. Perov. Simulation of peripheral vascular puncture control system. Biomedical Electronics, Moscow. (2014)

5. Kudashov I., S. Shchukin, O. Belaya, and S. Perov. Theoretical study of intravenous injection control system on heterogeneous model. Russian German Conference on Biomedical Engineering: Abstracts. St. Petersburg. 51-53 (2014).

6. Gabriel C., S. Gabriel, and E. Corthout. The dielectric properties of biological tissues: II. Literature survey. Phys. Med. Biol., 41:2231-2249, 1996. https://doi.org/10.1088/0031$9155 / 41 / 11 / 001$
7. AL-Harosh, M. B., and S. I. Shchukin. Numerical modeling of the electrical impedance method of peripheral veins localization. World Congress on Medical Physics and Biomedical Engineering. 51, 1683-1686 (2015).

8. Teschner, Eckhard, Michael Imhoff, and S. Leonhardt. Electrical Impedance Tomography: The realization of regional ventilation monitoring. Dräger Medical GmbH (2011).

9. Planken R. Nils, Xavier H. Keuter, Alfons G. Kessels, Arnold P. Hoeks, Tim Leiner, and Jan $\mathrm{H}$. Tordoir. Forearm cephalic vein cross-sectional area changes at incremental congestion pressures: towards a standardized and reproducible vein mapping protocol. J. Vasc. Surg. 44(2), 353-358 (2006). https://doi.org/10.1016/j.jvs.2006.04.038

10. Wilkinson, I. B. and Webb, D. J . Venous occlusion plethysmography in cardiovascular research: methodology and clinical applications. British J. Clin. Pharmacol. 55 631-646 (2001). https://doi.org/10.1046/j.0306-5251.2001.01495.x 